\title{
Selective implantation of diamines for cooperative catalysis in isoreticular heterometallic titanium-organic frameworks
}

\author{
Elena López-Maya, ${ }^{[a]}$ Natalia M. Padial, ${ }^{[a]}$ Javier Castells-Gil, ${ }^{[a]}$ Carolina R. Ganivet, ${ }^{[a]}$ Ana Rubio- \\ Gaspar, ${ }^{[a]}$ Francisco G. Cirujano, ${ }^{[a]}$ Neyvis Almora-Barrios, ${ }^{\text {[a] }}$ Sergio Tatay, ${ }^{[a]}$ Sergio Navalón ${ }^{[b]}$ and \\ Carlos Martí-Gastaldo*[a]
}

\begin{abstract}
We introduce the first example of isoreticular titanium-organic frameworks, MUV-10 and MUV-12, to show how the different affinity of hard $\mathrm{Ti}(\mathrm{IV})$ and soft $\mathrm{Ca}$ (II) metal sites can be used to direct selective grafting of amines to the last. This enables the combination of Lewis acid titanium centers and available $-\mathrm{NH}_{2}$ sites in two sizeable pores for cooperative cycloaddition of $\mathrm{CO}_{2}$ to epoxides at room temperature and atmospheric pressure. The selective grafting of molecules to heterometallic clusters adds up to the pool of methodologies available for controlling the positioning and distribution of chemical functions in precise positions of the framework required for definitive control of pore chemistry.
\end{abstract}

Metal-Organic Frameworks (MOFs) have arisen as promising materials for cooperative catalysis due to the combination of high surface areas, ${ }^{[1]}$ dense number of open metal sites (OMS) ${ }^{[2]}$ and crystalline structures for controllable arrangements of active sites and additional catalytic units. ${ }^{[3]}$ Cooperative catalysis relies on the acceleration of the reaction rate by simultaneous activation of two or more reactants by different catalytic sites. These processes are a common feature in enzymes, that have naturally evolved to make use of the active center and the surrounding environment for tuning their catalytic activity. ${ }^{[4,5]}$ Cooperativity in MOFs can be achieved from the interplay of open metal sites with organic cocatalysts, that can be incorporated to the solid either by using functionalized linkers during the synthesis ${ }^{[6-8]}$ or loaded postsynthetically as functional guests (Figure 1a). ${ }^{[9,10]}$ However, these strategies sometime offer poor control over the distribution of active sites in the solid or weak host-guest interactions detrimental to activity or recyclability. Chemical design can be used to circumvent these shortcomings and produce cooperative catalysts that feature a tailorable distribution of active sites by direct implantation in precise positions of the framework.

[a] Dr E.López-Maya, Dr N. M. Padial, Dr J. Castells-Gil, Dr C. R. Ganivet, Dr F. G. Cirujano, Dr. N. Almora-Barrios, Dr. S. Tatay and Dr C. Marti-Gastaldo

Functional Inorganic Materials team, Instituto de Ciencia Molecular, Universidad de Valencia. Catedrático Jose Beltrán-2, 46980, Paterna (Spain); e-mail: carlos.marti@uv.es

[b] Dr. S. Navalón

Departamento de Química, Universitat Politècnica de València, C/Camino de Vera, s/n, 46022, Valencia (Spain).

Supporting information for this article is given via a link at the end of the document.

Here, we have chosen a titanium-organic framework to demonstrate this concept. Despite MOFs often suffer from poor chemical stability that limits their use as heterogeneous catalysts the incorporation of hard, oxophilic $\mathrm{Ti}$ or $\mathrm{Zr}(\mathrm{IV})$ ions to their structure provides stronger bonds for exceptional stability in water. ${ }^{[11]}$ Although the challenging solution chemistry of titanium
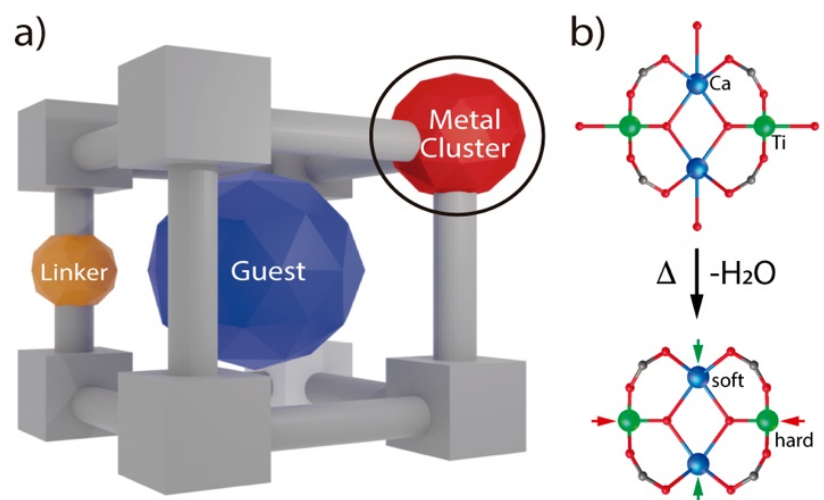

Figure 1. a) Different strategies to combined cooperative active sites in MOFs b) Creation of two types of open metal sites (hard and soft) generated by thermal treatment.

has limited the isolation of crystalline frameworks, the number of Ti-MOFs reported has increased significantly in the last years. ${ }^{[12-}$ ${ }^{16]}$ We recently reported the heterometallic MUV-10 framework $\left[\mathrm{TiV}_{3} \mathrm{Ca}_{3}{ }_{3}\left(\mu_{3}-\mathrm{O}\right)_{3}(\mathrm{btc})_{4}\left(\mathrm{H}_{2} \mathrm{O}\right)_{6}\right] \quad($ btc $=$ benzene-1,3,5tricarboxylate). ${ }^{[14]}$ This material combines excellent thermal and chemical stabilities with a surface area close to $1000 \mathrm{~m}^{2} \cdot \mathrm{g}^{-1}$, all relevant properties from a catalytic standpoint. In addition, it features tetranuclear heterometallic $\mathrm{Ti}_{2} \mathrm{Ca}_{2}$ clusters with axially coordinated water molecules that enable straightforward generation of two types of open metal sites (OMS) by thermal treatment.

We demonstrate how the different chemistry in the affinity of unsaturated hard $\mathrm{Ti}(\mathrm{IV})$ and soft $\mathrm{Ca}$ (II) Lewis acid sites to coordinate amines can be used for selective implantation of basic Brönsted sites to the last (Figure 1b). Compared to other routes based on encapsulation or impregnation, the generation of the cocatalyst is here controlled by the chemistry of the metal-oxo cluster and is thus compatible with the isoreticular expansion of the framework. To exemplify this advantage, we extend this method to MUV-12, a new material built from the interlinking of the same $\mathrm{Ti}_{2} \mathrm{Ca}_{2}$ with an extended tricarboxylic linker, that permits obtaining enlarged pores to generate a cooperative catalyst for quantitative cycloaddition of $\mathrm{CO}_{2}$ to epoxides at room temperature and atmospheric pressure that prevents the use of an additional molecular co-catalyst.

MUV-10 was synthesized according to the reported method. ${ }^{[14]}$ Phase purity was confirmed by chemical analysis and powder XRay diffraction (PXRD). We used water adsorption isotherms and FT-IR spectra of CO adsorption to confirm the creation of OMS by thermal treatment in vacuum (Figure S1 and S2). MUV-10 displays sodalite-type cages of $1.2 \mathrm{~nm}$ in diameter intersected in the $3 \mathrm{D}$ network by channels with $0.5 \mathrm{~nm}$ pore windows (Figure 2a), only compatible with the grafting of small diamines. Accordingly, we reacted desolvated MUV-10 crystals with 1,3diaminopropane (DAP) in anhydrous toluene for 12 hours at room 
temperature under inert atmosphere to produce MUV-10-DAP. This method is very similar to that used for appending diamines to the metal centres (Mg, Mn, $\mathrm{Zn}$ ) in MOF-74. ${ }^{[17,18]}$ The PXRD of MUV-10-DAP confirms that the appending of DAP is respectful with the structure of the framework (Figure 2b). The incorporation of the diamine in the solid is consistent with the presence of characteristic $\mathrm{C}-\mathrm{H}$ and $\mathrm{N}-\mathrm{H}$ amine vibrations at 2864 and 3280 respectively $\mathrm{cm}^{-1}$ in the FT-IR spectrum (Supplementary Section S2.2.4). The extent of DAP loading was evaluated with thermogravimetric analysis (TGA) and proton nuclear magnetic resonance ${ }^{1} \mathrm{H}$ NMR of the digested solid. Both suggest the grafting of approximately $13 \mathrm{wt} \%$ of DAP for the unit formula $\left[\mathrm{Ti}^{\mathrm{IV}}{ }_{3} \mathrm{Ca}_{3}{ }_{3}\left(\mu_{3}-\right.\right.$ $\left.\mathrm{O})_{3}(\mathrm{btc})_{4}(\mathrm{DAP})_{3}\right]$, consistent with the $\mathrm{CHN}$ analysis. Compared to homometallic $\mathrm{Mg}_{2}$ (dobpdc) that results in complete metal grafting for M:DAP ratios of $1: 1,{ }^{[19]}$ our experimental 2:1 ratio suggests that only $50 \%$ of the metal sites are grafted. This agrees well with a selective implantation to the soft $\mathrm{Ca}$ (II) sites in the heterometallic $\mathrm{Ti}_{2} \mathrm{Ca}_{2}$ cluster, more prone to interact with the primary amine of DAP than the hard oxophilic Ti(IV) metals. We next analyzed the changes in the porosity of the solid with $\mathrm{N}_{2}$ and $\mathrm{CO}_{2}$ adsorption experiments. Compared to MUV-10, the surface area of MUV-10DAP is reduced to close the $50 \%$ for a BET of $520 \mathrm{~m}^{2} \mathrm{~g}^{-1}$ (Figure 2c). This drop coincides with the reduction in free pore volume calculated with Materials Studio for a structural model of MUV-10 with DAP molecules appended only to $\mathrm{Ca}$ (II) sites (Table S1). $\mathrm{CO}_{2}$ isotherms also reveal an important decrease in gas uptake of close to $75 \%$ compared to MUV-10, but display a higher uptake in the low pressure region $(P<100$ Torr) indicative of a stronger interaction with $\mathrm{CO}_{2}$ (Figure S7). ${ }^{[20,21]}$ This is confirmed by the isosteric heat of $\mathrm{CO}_{2}$ adsorption of diamine appended MUV-10DAP $\left(61.3 \mathrm{~kJ} \mathrm{~mol}^{-1}\right)$ that almost triplicates the $27.7 \mathrm{~kJ} \mathrm{~mol}^{-1}$ of the pristine framework (Figure $\mathbf{2 d}$ ).

This experimental information suggested MUV-10-DAP might behave as a bifunctional heterogeneous catalyst from the combination of Lewis acid and available $-\mathrm{NH}_{2}$ sites. Before testing its ability to activate the cycloaddition of $\mathrm{CO}_{2}$ to epoxides in absence of a molecular co-catalyst, we deemed necessary to generate iodide ions that are known to activate ring opening. ${ }^{[22]}$ MUV-10-DAP crystals were suspended in dry $\mathrm{CH}_{3} \mathrm{CN}$ and reacted with methyl iodide $\left(\mathrm{CH}_{3} \mathrm{l}\right)$ at $80^{\circ} \mathrm{C}$ for $48 \mathrm{~h}$ under $\mathrm{N}_{2}$ atmosphere according to a reported method. ${ }^{[22]}$ We evaluated the incorporation of iodine by ICP analysis (Supplementary Section S2.1). Unfortunately, our results discarded a successful generation of iodine ions. This is likely due to the strict limitations imposed by the grafting of DAP to the pore openings and empty space available in MUV-10, which is quite small in origin (Figure 2e and Table S1).
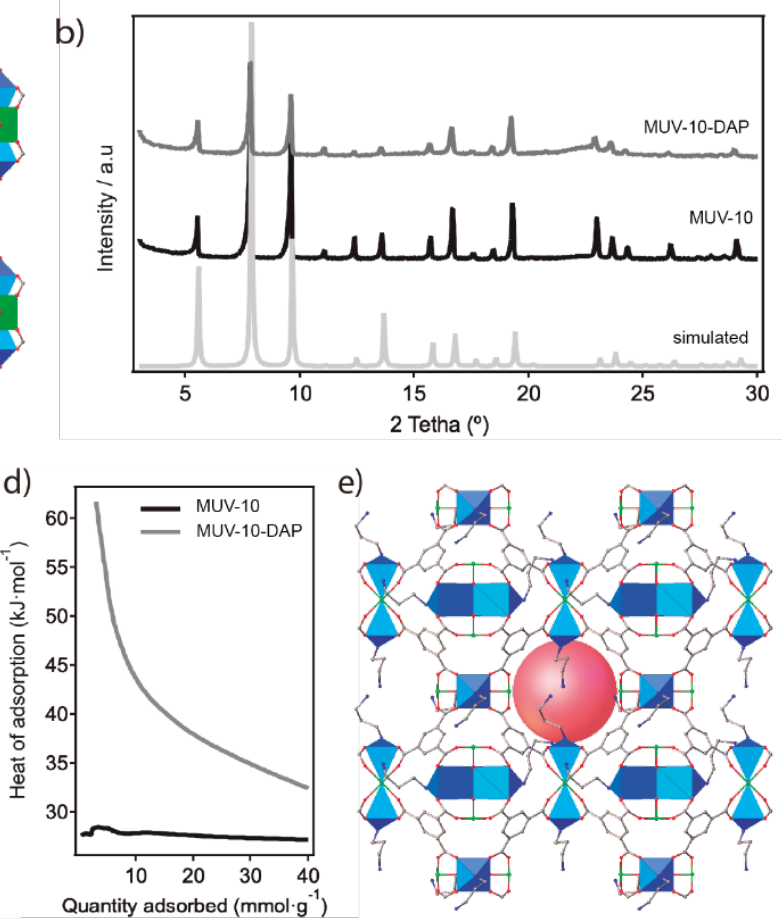

Figure 2. a) Structure of MUV-10 featuring sodalite-type octahedral cages of $1.2 \mathrm{~nm}$ in diameter (lavender sphere). b) PXRD patterns of MUV-10 and MUV-10-DAP after reaction with the diamine. c) $\mathrm{N}_{2}$ isotherms showing the drop of surface area as result of the incorporation of DAP (MUV-10-DAP), which also induces a drastic increase in the d) $\mathrm{CO}_{2}$ isosteric heat of adsorption. e) The simulated structure of MUV-10-DAP reveals a reduction

This limitation encouraged us to synthesize an isoreticular framework that combined superior porosity and heterometallic clusters amenable to selective DAP grafting. The reticulation of organic linkers of the same geometry and connectivity but variable size permits controlling the metrics and functionality of a targeted framework topology, that is in turn determined by the structural information encoded in the inorganic node or secondary building unit (SBU). ${ }^{[23]}$ Reticular chemistry has been incredibly fruitful in the design of multiple MOFs ${ }^{[24]}$ but the synthetic difficulties inherent to the chemistry of titanium in solution have neglected the discovery of persistent SBUs for the synthesis of isoreticular Ti-MOFs thus far. We synthesized 3,5-tris(4carboxyphenyl)benzene $\left(\mathrm{H}_{3} \mathrm{btb}\right)$ according to the reported protocol (Figure 3a, Supplementary Section S3.1). Reaction of $\mathrm{H}_{3}$ btb with calcium chloride and titanium(IV) isopropoxide at 120 ${ }^{\circ} \mathrm{C}$ in $\mathrm{N}, \mathrm{N}$-dimethylformamide (DMF) by using acetic acid as modulator yields colourless, octahedral crystals of ca. $25 \mu \mathrm{m}$ in size (Figure $\mathbf{3 b}$ ). Single-crystal XRD measurements revealed that MUV-12 $\left[\mathrm{Ti}^{\prime V} \mathrm{Ca}_{3}{ }_{3}\left(\mu_{3}-\mathrm{O}\right)_{3}(\mathrm{btb})_{4}\left(\mathrm{H}_{2} \mathrm{O}\right)_{6}\right]$ is a doubly interpenetrated framework isoreticular to MUV-10. It crystallizes in the cubic space group / $m$-3 ( $\mathrm{a}=26.4010 \AA)$ and, despite interpenetration, doubles the accessible void space with pore diameters of $2.4 \mathrm{~nm}$ (Figure 3c). Besides overcoming the spatial limitations intrinsic to MUV-10, we argued framework interpenetration might be beneficial from a catalytic standpoint due to a higher density of active sites and enhanced robustness. ${ }^{[25]}$ 

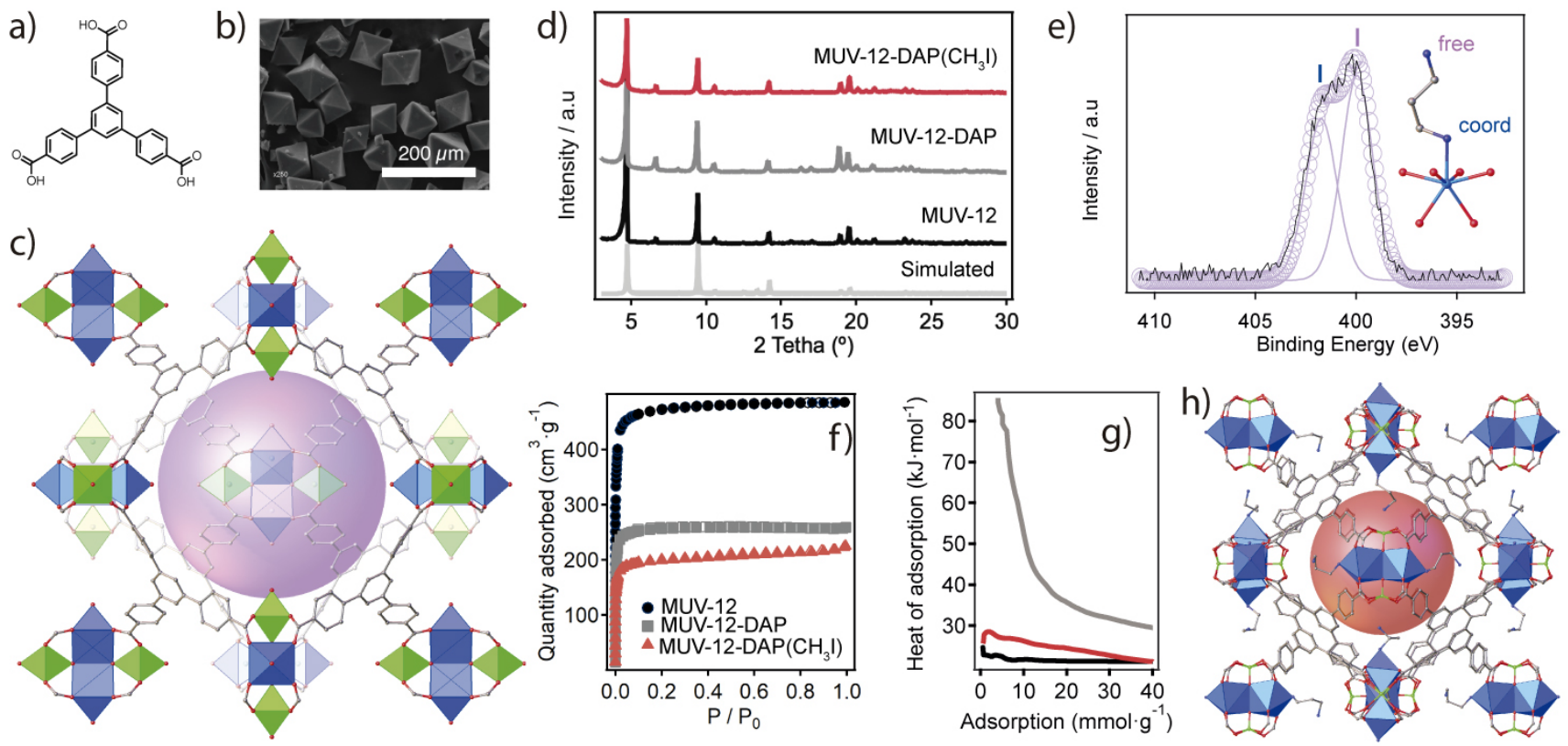

Figure 3. a) Structure of $\mathrm{H}_{3}$ btb linker. b) SEM images of MUV-12 crystals. c) Structure of MUV-12 featuring an interpenetrated structure and sodalite-type octahedral cages of ca. $2.2 \mathrm{~nm}$ in diameter (lavender sphere). d) PXRD patterns of MUV-12, MUV-12-DAP and MUV-12-DAP(CH $\mathrm{C}_{3}$ ). e) XPS of the N 1s region in MUV-12-DAP f) $\mathrm{N}_{2}$ isotherms showing the drop of surface area as result of the incorporation of DAP (MUV-12-DAP) as well as the incorporation of iodide ions $\left(\mathrm{MUV}-12-\mathrm{DAP}\left(\mathrm{CH}_{3} \mathrm{I}\right)\right.$ ) g) $\mathrm{CO}_{2}$ isosteric heat of adsorption of MUV-12, MUV-12-DAP and MUV-12-DAP( $\left.\mathrm{CH}_{3} \mathrm{I}\right)$. h) Simulated pore structure of MUV-12-DAP reveals a reduction in the pore windows and internal cavity (red sphere) of close to $50 \%$ that limits substrate uptake.

We synthesized a fresh batch of MUV-12 and confirmed phase purity by PXRD and CHN (Supplementary Section S3), followed by DAP grafting and thorough washing according to the same method used for MUV-10. It is worth noticing that all materials used in this work were subjected to a thermal treatment to prevent the presence of uncoordinated diamine in the pore as confirmed by X-Ray Photoelectron Spectroscopy (XPS, Supplementary Figure S11). TGA and ${ }^{1} \mathrm{H}$ NMR suggest the appending of approximately 7.8 weight percent $(w t \%)$ of DAP to the MUV-12 solid for a M:DAP ratio of $2: 1$. This value is equivalent to that obtained for MUV-10 and further supports the selective implantation to $\mathrm{Ca}(\mathrm{II})$ sites for the formation of $\left[\mathrm{TiV}^{\mathrm{IV}} \mathrm{Ca}_{3} \mathrm{Cl}_{3}\left(\mu_{3^{-}}\right.\right.$ $\left.\mathrm{O})_{3}(\mathrm{btb})_{4}(\mathrm{DAP})_{3}\right]$. This unit formula was also corroborated by $\mathrm{CHN}$ analysis of the crystals. It is worth noting that MUV-12 does not suffer from the limitations to the diffusion and grafting of the diamine imposed by MUV-10. As result, it could incorporate more easily a stochiometric M:DAP ratio unless a marked difference in the affinity of both metals for the diamine coordination exists. As supported by MUV-12-DAP PXRD data, DAP grafting has a negligible impact on the structure of MUV-12 (Figure 3d). We used nitrogen 1s X-ray photoelectron spectroscopy (XPS) to confirm the appending of the diamine to the framework. Figure 3e shows the N1s high-resolution XPS spectrum of MUV-12-DAP. The N1s signal confirms the presence of nitrogen and reveals two different chemical environments in roughly $1: 1$ ratio. The contribution centered at 400.0 and $402.3 \mathrm{eV}$ can be attributed to free and coordinated to the metal amine groups, respectively. ${ }^{[26,27]}$. The effect of DAP grafting on the porosity of MUV-12 is quite similar to that observed for MUV-10. As shown in Figure 3f, the experimental BET surface area of $2100 \mathrm{~m}^{2} \cdot \mathrm{g}^{-1}$ in MUV-12 drops to around $50 \%$ in MUV-12-DAP $\left(980 \mathrm{~m}^{2} \cdot \mathrm{g}^{-1}\right)$. The analysis of $\mathrm{CO}_{2}$ isotherms at 273,283 and $293 \mathrm{~K}$ reveals that MUV-12-DAP outperforms the non-aminated framework at low-P regimes, indicative of enhanced adsorption (Supplementary Figure S14). This is consistent with the drastic increase in the isosteric heat of $\mathrm{CO}_{2}$ adsorption $\left(Q_{s t}\right)$ observed after appending of DAP (Figure 3g). MUV-12-DAP $Q_{s t}$ of $84.6 \mathrm{~kJ} \mathrm{~mol}^{-1}$, reaches close to four times the value of pristine MUV-12 (24.3 kJ mol-1).

The simulated structural model of MUV-12-DAP reveals a free volume of $2176 \AA^{3}$ with accessible pore diameters of close to 20 $\AA$ and pore windows of $7 \AA$ (Figure $3 \mathrm{~h}$ ). Compared to the spatial limitations intrinsic to MUV-10-DAP, this value shall enable generation of nucleophilic iodide anions in the pore more easily. Accordingly, we reacted MUV-12-DAP crystals with $\mathrm{CH}_{3}$ l by following the same procedure described above for its isoreticular counterpart. The resulting material MUV-12-DAP $\left(\mathrm{CH}_{3} \mathrm{I}\right)$ was analyzed with ICP (Supplementary Section S3.1) and energy dispersive X-ray (EDX) to confirm the generation of iodine ions. In addition, mapping images show the homogeneous distribution of iodine across the crystal and scanning electronic microscopy (SEM) images verify that the size and morphology of the crystals is maintained in MUV-12-DAP $\left(\mathrm{CH}_{3} \mathrm{l}\right)$ discarding significant damage (Figure Supplementary S16). Moreover, the PXRD of MUV-12-DAP $\left(\mathrm{CH}_{3} \mathrm{l}\right)$ confirms that the framework can survive multistep post-synthetic modification (PSM) with negligible changes to the original diffraction pattern (Figures 3d and S10). Nonetheless, $\mathrm{CHN}$ analysis reveal that this PSM induces a loss of close to $60 \%$ of the nitrogen originally present in MUV-12-DAP. The incorporation of iodine does not lead to a high decrease in BET surface area value $\left(850 \mathrm{~m}^{2} \cdot \mathrm{g}^{-1}\right)$ for MUV-12-DAP (Figure $\left.3 \mathrm{f}\right)$, this is due to the sublimation of iodine during the activation of the framework prior to gas adsorption, as confirmed by the EDX analysis of the material after the isotherm that reveals a complete loss of iodine (Figure Supplementary S16e). By contrast, the treatment with $\mathrm{CH}_{3}$ l reduced $\mathrm{CO}_{2}$ uptake at $20^{\circ} \mathrm{C}\left(40 \mathrm{~cm}^{3} \cdot \mathrm{g}^{-1}\right)$. The weaker interaction with $\mathrm{CO}_{2}$ denoted by the absence of steep increases in the gas uptake at low-P indicate the modification of $\mathrm{NH}_{2}$ groups (Figure Supplementary S15). This is further corroborated by the decrease of the heat of adsorption at zero 

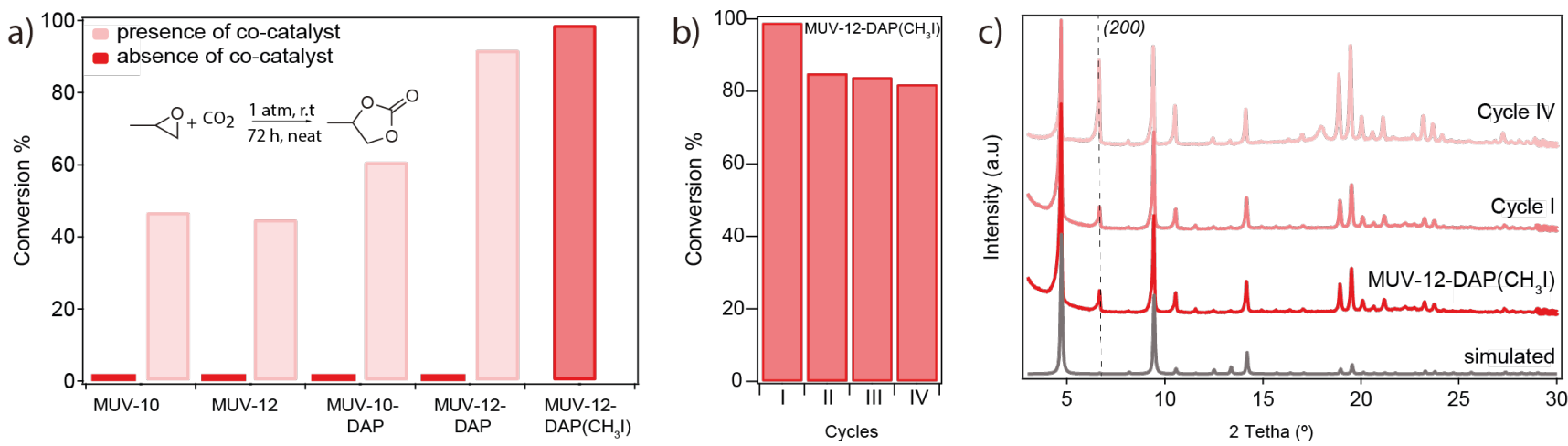

Figure 4. a) Cyclic carbonate conversion though cycloaddition of $\mathrm{CO}_{2}$ into propylene oxide using MUV-10, MUV-12 MUV-10-DAP and MUV-12-DAP in presence $(8 \% \mathrm{~mol})$ and in absence of co-catalyst and MUV12-DAP $\left(\mathrm{CH}_{3} \mathrm{l}\right)$ only in absence of co-catalyst. b) Recycled experiments of cycloaddition of $\mathrm{CO} 2$ with propylene oxide catalysed by MUV-12-DAP $\left(\mathrm{CH}_{3} \mathrm{I}\right)$. c) PXRD of MUV-12 simulated, MUV-12-DAP $\left(\mathrm{CH}_{3} \mathrm{I}\right)$, MUV-12-DAP $\left(\mathrm{CH}_{3} \mathrm{I}\right)$ after the first cycle and MUV-12-DAP(CH $\left.\mathrm{I}\right)$ after fourth cycle of catalysis.

coverage to $28.4 \mathrm{~kJ} \mathrm{~mol}^{-1}$ (Figure $\mathbf{3 g}$ ), slightly above pristine MUV-12.

To illustrate the value of diamine grafting and incorporation of halides to the framework, we tested the catalytic activity of the MUV-10 and MUV-12 families in the formation of cyclic carbonates by the cycloaddition of $\mathrm{CO}_{2}$ to epoxides. There are previous reports on the use of MOFs to catalyze this reaction but they generally require high pressures, high temperatures or the use of a co-catalyst. ${ }^{[22,28-30]}$ This renders a complicated scenario that is not ideal from an economical and environmental standpoint. The design of more efficient catalysts capable of $\mathrm{CO}_{2}$ conversions at room temperature and atmospheric pressure without a co-catalyst is highly desirable. To demonstrate the efficiency of our material, we tested the cycloaddition of $\mathrm{CO}_{2}$ to form propylene carbonate at room temperature and $1 \mathrm{~atm}$ of pressure (Figure 4a). The conversion with pristine MUV-10 and MUV-12 is negligible in absence of a co-catalyst, whereas addition of tetrabutylammonium bromide (TBAB) leads conversions slightly above $40 \%$ in both materials. We do not observe significant differences in conversion that could be attributed to the superior porosity of the extended MUV-12 framework. More clear differences can be observed for the changes in catalytic activity after the appending of DAP. Just like their non-aminated counterparts, MUV-10-DAP and MUV-12DAP are inactive in absence of TBAB but display comparatively higher conversions after the addition of the co-catalyst. This boost is particularly significant for MUV-12-DAP, that reaches close to $90 \%$ in conversion compared to the $60 \%$ of MUV-10-DAP. We argue these changes between isostructural frameworks reveal the positive effect of $-\mathrm{NH}_{2}$ Brönsted sites to activate $\mathrm{CO}_{2}$ for the formation of the corresponding cyclic carbonate. ${ }^{[31]}$ This effect is more acute for MUV-12 possibly from its superior porosity area and interpenetrated structure. This combination results in a superior $\mathrm{CO}_{2}$ uptake at $20^{\circ} \mathrm{C}\left(46 \mathrm{~cm}^{3} \cdot \mathrm{g}^{-1}\right)$ and a higher density of appended DAP molecules with the associated increase in $\mathrm{Q}_{\mathrm{st}}$ for more efficient $\mathrm{CO}_{2}$ activation. Finally, MUV-12-DAP $\left(\mathrm{CH}_{3} \mathrm{l}\right)$ displays the highest efficiency of the series and is the only material that reaches complete conversion in absence of the molecular co-catalyst. It should be noted that for a control physical mixture of MUV-12, DAP and $\mathrm{CH}_{3}$ l the conversion obtained was only $40 \%$, thus suggesting that the implantation of DAP plays a key role for the subsequent generation of iodide ions inside the pore in MUV-12-DAP $\left(\mathrm{CH}_{3} \mathrm{l}\right)$ making unnecessary the use of an additional co-catalyst to complete the reaction. MUV-12$\operatorname{DAP}\left(\mathrm{CH}_{3} \mathrm{l}\right)$ behaves as a cooperative catalyst from the combination of Lewis acid Ti(IV) and nucleophilic iodide anions, both cooperating in the pores of the framework for synergetic interaction in a confined space. The established mechanism for this reaction involves a Lewis acid site to activate the epoxide towards the nucleophilic attack by the halide (Scheme S1). Whereas the density of Lewis sites is fixed by the heterometallic $\mathrm{Ti}_{2} \mathrm{Ca}_{2}$ cluster, our method permits producing MUV-12-DAP $\left(\mathrm{CH}_{3} \mathrm{I}\right)$ catalysts with variable amounts of nucleophile to evaluate the effect of these iodide anions on the overall performance. By changing the temperature and reaction time used for the synthesis of MUV-12-DAP $\left(\mathrm{CH}_{3} \mathrm{l}\right)$ (Table S4), we synthesized materials with different amounts of iodine. The catalytic tests confirm that epoxide conversion in our case is controlled by the nucleophile, in good agreement with the poor efficiencies encountered for MUV-10-DAP and MUV-12-DAP in absence of TBAB.

To put our results in a general context, we have summarized the catalytic activity reported for other MOFs used for the transformation of epoxides into cyclic carbonates at standard pressure (Table Supplementary S5). MUV-12-DAP $\left(\mathrm{CH}_{3} \mathrm{l}\right)$ is the only one that displays quantitative conversion at room temperature, just comparable to the activity displayed by composites of mesoporous MIL-101 or PCN-333 prepared by infiltration with an ionic polymer. ${ }^{[10]}$ Compared to this approach that might induce the co-precipitation of the polymer at the surface of the crystals, our method enables direct implantation of the cooperating sites in the confined space by using the chemistry of the SBU. To investigate if the $\mathrm{CO}_{2}$ transformation was ascribed exclusively to in-pore reactivity, we also tested the same reaction under equivalent conditions with other substrates of increasing sizes (Table Supplementary S5). We observe a progressive decrease with the size of the substrate down to $30 \%$ in conversion for 1,2-epoxybutane, $7 \%$ for butyl glycidyl ether and below $3 \%$ for bigger substrates, suggesting in-pore reactivity, although some other electronic effects linked to the structure of the epoxides cannot by fully discarded. Also important, the absence of the need for any additional component simplifies the recovery of the catalyst after the reaction. Figure $\mathbf{4 b}$ shows the cyclability of MUV-12-DAP $\left(\mathrm{CH}_{3} \mathrm{l}\right)$, which maintains the conversion of propylene carbonate above $80 \%$ upon four consecutive cycles. According to the PXRD of the material after each test (Figure 4c) this small loss in activity is not indicative of the degradation of the framework. This is more likely due to the blocking of the pores by the product of the reaction in view of the changes in relative 
intensity of the diffraction peaks in accordance with different loadings of the MUV-12-DAP $\left(\mathrm{CH}_{3} \mathrm{l}\right)$ pores. ${ }^{\text {[2] }}$

We have introduced here the first family of isoreticular titanium frameworks, MUV-10 and MUV-12, to demonstrate the ability of heterometallic $\mathrm{Ti}_{2} \mathrm{Ca}_{2}$ clusters to direct the selective implantation of diamines to the soft $\mathrm{Ca}(\mathrm{II})$ sites in the inorganic node. This results in the combination of Lewis acid Ti(IV) centers and available $-\mathrm{NH}_{2}$ sites. Chemical modification of MUV-12-DAP with $\mathrm{CH}_{3}$ l enables quantitative cycloaddition of $\mathrm{CO}_{2}$ to propylene oxide at room temperature and atmospheric pressure for complete conversion in absence of additional co-catalysts and with excellent cyclability. This selective grafting of molecules to heterometallic clusters adds up to the pool of methodologies available for controlling the positioning and distribution of functional moieties in MOFs. We are confident the combined use of the empty space offered by these porous frameworks, alongside the different chemistries intrinsic to metal clusters and organic linkers, offers a promising playground for producing chemically complex environments by implantation of multiple components for unprecedented opportunities in synergistic phenomena. ${ }^{[33]}$

\section{Acknowledgements}

This work was supported by the EU (ERC Stg Chem-fs-MOF 714122) and Spanish Government (CTQ2017-83486-P, RTI2018-098568-A-I00 \& CEX2019-000919-M). E.L.-M. and S.T. thanks the Spanish Government for their Juan de la Cierva Fellowship (FJCl-2017-32956) and Ramon y Cajal contract (RYC2016-1981), respectively. N.M.P. thanks the European Union for a Marie Skłodowska-Curie Global Fellowship (H2020-MSCA-IF2016-GF-749359-EnanSET). F. G. C. acknowledges the support of a fellowship from "la Caixa" Foundation (ID 100010434). The fellowship code is LCF/BQ/PI19/11690011. S.N. thanks financial support by the Ministerio de Ciencia, Innovación y Universidades (RTI 2018-099482-A-I00 project), Fundación Ramón Areces (XVIII Concurso Nacional para la Adjudicación de Ayudas a la Investigación en Ciencias de la Vida y de la Materia, 2016) and Agencia Valenciana de la Innovación (AVI-GVA, Carboagua project, INNEST/2020/111).

Keywords: titanium-organic frameworks $\cdot$ carbon dioxide conversion $\cdot$ cooperative catalysis $\bullet$ isoreticular chemistry • heterometallic cluster

Z. Chen, P. Li, R. Anderson, X. Wang, X. Zhang, L. Robison, L. R Redfern, S. Moribe, T. Islamoglu, D. A. Gómez-Gualdrón, T. Yildirim, J. F. Stoddart, O. K. Farha, Science. 2020, 368, 297-303 Y.S. Wei, M. Zhang, R. Zou, Q. Xu, Chem Rev, 2020, 120,12089 12174

[3] Y. B. Huang, J. Liang, X. S. Wang, R. Cao, Chem. Soc. Rev. 2017, $46,126-157$

[4] X. H. Matthew D. Wodrich, Nat. Rev. Chem. 2017, 2, 1-7.

[5] B. Le Bailly, Nat. Rev. Chem. 2018, 2, 385-397.

[6] P. Z. Li, X. J. Wang, J. Liu, J. S. Lim, R. Zou, Y. Zhao, J. Am. Chem Soc. 2016, 138, 2142-2145.

[7] C. Li, H. Tang, Y. Fang, Z. Xiao, K. Wang, X. Wu, H. Niu, C. Zhu, H. C. Zhou, Inorg. Chem. 2018, 57, 13912-13919.

[8] B. Li, K. Leng, Y. Zhang, J. J. Dynes, J. Wang, Y. Hu, D. Ma, Z. Shi, L. Zhu, D. Zhang,Y. Sun, M. Chrzanowsk, S. Ma, J. Am. Chem. Soc. 2015, 137, 4243-4248.

[9] X. Li, B. Zhang, L. Tang, T. W. Goh, S. Qi, A. Volkov, Y. Pei, Z. Qi, C. K. Tsung, L. Stanley, W. Huang, Angew. Chem. Int. Ed. 2017, 56, 16371-16375.
[10] B. Aguila, Q. Sun, X. Wang, E. O'Rourke, A. M. Al-Enizi, A. Nafady, S. Ma, Angew. Chem. Int. Ed. 2018, 57, 10107-10111.

[11] A. J. Howarth, Y. Liu, P. Li, Z. Li, T. C. Wang, J. T. Hupp, O. K. Farha, Nat. Rev. Mater. 2016, 1, 1-15.

[12] N. M. Padial, B. Lerma-Berlanga, N. Almora-Barrios, J. Castells-Gil, I. da Silva, M. de la Mata, S. I. Molina, J. Hernández-Saz, A. E. Platero-Prats, S. Tatay, C. Marti-Gastaldo., J. Am. Chem. Soc. 2020 142, 6638-6648.

[13] J. Castells-Gil, N. M. Padial, N. Almora-Barrios, I. Da Silva, D. Mateo, J. Albero, H. García, C. Martí-Gastaldo, Chem. Sci. 2019, 10, 43134321.

[14] J. Castells-Gil, N. M. Padial, N. Almora-Barrios, J. Albero, A. R. RuizSalvador, J. González-Platas, H. García, C. Martí-Gastaldo, Angew. Chem Int. Ed. 2018, 57, 8453-8457.

[15] L. Zou, D. Feng, T. F. Liu, Y. P. Chen, S. Yuan, K. Wang, X. Wang, S. Fordham, H. C. Zhou, Chem. Sci. 2016, 7, 1063-1069.

[16] H. L. Nguyen, F. Gándara, H. Furukawa, T. L. H. Doan, K. E. Cordova, O. M. Yaghi, J. Am. Chem. Soc. 2016, 138, 4330-4333.

[17] P. J. Milner, R. L. Siegelman, A. C. Forse, M. I. Gonzalez, T. Runčevski, J. D. Martell, J. A. Reimer, J. R. Long, J. Am. Chem. Soc 2017, 139, 13541-13553

[18] C. M. McGuirk, R. L. Siegelman, W. S. Drisdell, T. Runčevski, P. J. Milner, J. Oktawiec, L. F. Wan, G. M. Su, H. Z. H. Jiang, D. A. Reed M. I. Gonzalez, D. Prendergast, J. R. Long., Nat. Commun. 2018, 9, $1-10$.

[19] P. J. Milner, R. L. Siegelman, A. C. Forse, M. I. Gonzalez, T. Runčevski, J. D. Martell, J. A. Reimer, J. R. Long, J. Am. Chem. Soc 2017, 139, 13541-13553.

[20] C. Montoro, E. García, S. Calero, M. A. Pérez-Fernández, A. L. López, E. Barea, J. A. R. Navarro, J. Mater. Chem. 2012, 22, $10155-$ 10158.

[21] A. Demessence, D. M. D'Alessandro, M. L. Foo, J. R. Long, J. Am. Chem. Soc. 2009, 131, 8784-8786.

[22] J. Liang, R. P. Chen, X. Y. Wang, T. T. Liu, X. S. Wang, Y. B. Huang, R. Cao, Chem. Sci. 2017, 8, 1570-1575

[23] M. J. Kalmutzki, N. Hanikel, O. M. Yaghi, Sci. Adv. 2018, 4, 1-16.

[24] M. Eddaoudi, J. Kim, N. Rosi, D. Vodak, J. Wachter, M. O'Keeffe, O. M. Yaghi, Science 2002, 295, 469-472.

[25] Z. Lin, Z. M. Zhang, Y. S. Chen, W. Lin, Angew. Chem. Int. Ed. 2016, $55,13739-13743$.

[26] W. Cao, Z. Wang, Q. Zeng, C. Shen, Appl. Surf. Sci. 2016, 389, 404410.

[27] J. Ederer, P. Janoš, P. Ecorchard, J. Tolasz, V. Štengl, H. Beneš, M. Perchacz, O. Pop-Georgievski, RSC Adv. 2017, 7, 12464-12473.

[28] J. Zhu, P. M. Usov, W. Xu, P. J. Celis-Salazar, S. Lin, M. C. Kessinger, C. Landaverde-Alvarado, M. Cai, A. M. May, C. Slebodnick, D. Zhu, S. D. Senanayake, A. J. Morris, J. Am. Chem. Soc. 2018, 140, 993-1003.

[29] H. He, Q. Sun, W. Gao, J. A. Perman, F. Sun, G. Zhu, B. Aguila, K. Forrest, B. Space, S. Ma, Angew. Chem .Int. Ed. 2018, 57, 46574662.

[30] C. Huang, J. Dong, W. Sun, Z. Xue, J. Ma, L. Zheng, C. Liu, X. Li, K. Zhou, X. Qiao, Q. Song, W. Ma, L. Zhang, Z. Lin, T. Wang, Nat. Commun. 2019, 10, 1-10.

[31] Q. Han, B. Qi, W. Ren, C. He, J. Niu, C. Duan, Nat. Commun. 2015, 6, 1-8.

[32] H. S. Cho, J. Yang, X. Gong, Y. B. Zhang, K. Momma, B. M. Weckhuysen, H. Deng, J. K. Kang, O. M. Yaghi, O. Terasaki, Nat. Chem. 2019, 11, 562-570.

[33] J. Castells-Gil, N. M. Padial, N. Almora-Barrios, R. Gil-San-Millán, M. Romero-Ángel, V. Torres, I. da Silva, B. C. J. Vieira, J. C. Waerenborgh, J. Jagiello, J. A. R. Navarro, S. Tatay, C. MartiGastaldo, Chem 2020, 6, 3118-3131. 


\section{Disclamer}

This document is the unedited Author's version of a Submitted Work that was subsequently accepted for publication in Angewandte Chemie International Edition, copyright (C) Wiley-VCH on behalf of the German Chemical Society after peer review.

To access the final edited and published work see: https://doi.org/10.1002/anie.202100176 